\title{
Dynamic stall control over a rotor airfoil based on AC DBD plasma actuation
}

\author{
Guangyin Zhao ${ }^{1,2^{*}} \mathbb{D}$, Yong Huang ${ }^{2}$, Yongdong Yang ${ }^{2}$, Guoqiang $\mathrm{Li}^{2}$ and Hesen Yang ${ }^{3}$
}

\author{
* Correspondence: zym19860615@ \\ 163.com \\ ${ }^{1}$ State Key Laboratory of \\ Aerodynamics, China Aerodynamics \\ Research and Development Center, \\ Mianyang 621000, China \\ ${ }^{2}$ Low Speed Aerodynamics Institute, \\ China Aerodynamics Research and \\ Development Center, Mianyang \\ 621000, China \\ Full list of author information is \\ available at the end of the article
}

\begin{abstract}
At present, the control capability of dielectric barrier discharge (DBD) plasma actuation covers the flow velocity range of helicopter's retreating blades, so it is necessary to extend it to the dynamic stall control of rotor airfoils. A DBD plasma actuator was adopted to control the dynamic stall of an oscillating CRA309 airfoil in this paper. The effectiveness of alternating current (AC) DBD plasma actuation on reducing the area of lift hysteresis loop of the oscillating airfoil was verified through pressure measurements at a Reynolds number of $5.2 \times 10^{5}$. The influence of actuation parameters on the airfoil's lift and moment coefficients was studied. Both steady and unsteady actuation could effectively reduce the hysteresis loop area of the lift coefficients. The flow control effect of dynamic stall was strongly dependent on the history of angle of attack. Compared with the steady actuation, unsteady actuation had more obvious advantages in dynamic stall control, with reducing the area of lift hysteresis loop by more than 30\%. The effects of plasma actuation on the airfoil's flow field at both upward and downward stages were discussed at last.
\end{abstract}

Keywords: Plasma actuation, Flow separation, Dynamic stall, Hysteresis, Dielectric barrier discharge

\section{Introduction}

Dynamic stall phenomenon widely exists on helicopter's retreating blades and highly maneuverable aircraft. Dynamic stall can cause the wing's flutter with a sharp drop in lift and a rapid increase in drag and moment. For helicopters, the most direct impact of the blade's dynamic stall is to limit its maximum forward speed. Therefore, the study of dynamic stall control is of great engineering significance. Plasma actuation is prone to cause rapid and controllable broadband pneumatic actuation, and has a potential in dynamic stall control. Common plasma actuation methods include plasma synthetic jets (PSJ) [1], surface arc discharge (SAD) [2, 3] and dielectric barrier discharge (DBD) [4]. PSJ and SAD actuations are mostly used for shock wave control in the supersonic fields, while the low-speed flow separation is usually controlled by plasma of AC DBD and pulsed nanosecond surface (NS) DBD [5]. The research progress of DBD plasma actuation in flow control was summarized in Literature [6].

Flow separation control by DBD plasma actuation is mainly focused on static airfoils; while the dynamic stall control of oscillating airfoils has not been widely studied.

(c) The Author(s). 2021 Open Access This article is licensed under a Creative Commons Attribution 4.0 International License, which permits use, sharing, adaptation, distribution and reproduction in any medium or format, as long as you give appropriate credit to the original author(s) and the source, provide a link to the Creative Commons licence, and indicate if changes were made. The images or other third party material in this article are included in the article's Creative Commons licence, unless indicated otherwise in a credit line to the material. If material is not included in the article's Creative Commons licence and your intended use is not permitted by statutory regulation or exceeds the permitted use, you will need to obtain permission directly from the copyright holder. To view a copy of this licence, visit http://creativecommons.org/licenses/by/4.0/. 
Dynamic stall control by DBD plasma actuation started about 15 years ago [7]. The literature in recent years has an increasing trend $[8,9]$, indicating that dynamic stall control by plasma actuation has begun to attract more attention. Yang HS et al. [10] summarized the airfoil's dynamic stall control by plasma actuation in recent years. For helicopters, the typical flow velocity over the retreating blades is on the order of $100 \mathrm{~m} /$ $\mathrm{s}(\mathrm{Ma}=0.3)$ and the Reynolds number is on the order of $10^{6}$ [11]. This typical flow condition is within the controllable range of DBD plasma actuation. DBD plasma actuation is expected to provide a new control method for improving the rotor's performance during dynamic stall.

Mitsuo K et al. [12] found that the pulsed AC DBD could improve the lift hysteresis loop at the optimal actuation frequency of $F^{+}=0.5$ at the free-stream velocity $10 \mathrm{~m} / \mathrm{s}$ $50 \mathrm{~m} / \mathrm{s}$. Frankhouser M et al. [13] found that NS DBD actuation could improve stall state and promote the early recovery of wing's upper surface pressure. Sekimoto $\mathrm{S}$ et al. [14] found that the separated flow can be reattached under actuation during the down phase of an oscillating NACA0015 airfoil. Yu HC and Zheng JG found that NS DBD achieves surprising success in enhancing lift and reducing aerodynamic hysteresis at

$R e=7.5 \times 10^{5}$, whereas AC DBD nearly has no effect on the flow [15]. The influence of NS-DBD actuation parameters on the dynamic stall control effect was investigated with the freestream velocity $U_{\infty}=50 \mathrm{~m} / \mathrm{s}$ in paper [16]. It is found that the flow control effect can be maximized by adopting the low nondimensional actuation frequency. Paper [17] numerically revealed that $F^{+}=50$ is the most effective for delaying dynamic stall onset, $F^{+}=0.5$ is best for enhancing aerodynamic forces during full stall, and $F^{+}=6$ is best for promoting reattachment. Lombardi AJ [18] revealed that the pulsed AC DBD actuation could increase the integrated lift of an oscillating airfoil by $12 \%$ and reduce the maximum nose-down moment by $60 \%$.

However, the influence law of discharge parameters is still not very clear, in particular, the result of the optimal dimensionless actuation frequency is not consistent in these researches. Most studies use symmetrical airfoil of NACA series, such as NACA0015 or NACA0012 airfoil. It is more meaningful to choose an advanced practical airfoil of helicopter blade in the research. The reduced frequency in most studies is less than 0.1. Also, the control effect of AC DBD needs to be further evaluated. In this paper, at the free-stream velocity of $U_{\infty}=25 \mathrm{~m} / \mathrm{s}$, with corresponding chord-based Reynolds number of $5.2 \times 10^{5}$, the discharge voltage, discharge frequency, modulation frequency and duty cycle of unsteady AC DBD actuation were changed in the wind tunnel tests to evaluate the flow control effect. Time-resolved pressure measurement was conducted to obtain flow control effect by AC DBD plasma actuation. The effects of plasma actuation on the airfoil's flow field at both upward and downward stages were also discussed at last.

\section{Experimental setup}

The test was completed in the FL-11 wind tunnel at China Aerodynamics Research and Development Center (CARDC). The size of the test section is $1.8 \mathrm{~m}$ (width) $\times 1.4 \mathrm{~m}$ (height). The stable wind speed ranges from $10 \mathrm{~m} / \mathrm{s}$ to $105 \mathrm{~m} / \mathrm{s}$. The turbulence is less than 0.0008 when the wind speed is lower than $70 \mathrm{~m} / \mathrm{s}$.

A China's 3rd generation rotor airfoil (CRA309) was used in the test. The CRA309 is an advanced rotor airfoil developed by CARDC, and its maximum relative thickness is 
9\% of chord length. The airfoil made of Carbon fiber had a span length of $1.78 \mathrm{~m}$ and a chord length of $0.4 \mathrm{~m}$. The pitching motion was realized using an upgraded experimental setup driven by a servo motor. The oscillating frequency of the airfoil could be varied from 0.1-5 Hz. During the current test, the CRA309 airfoil was oscillated periodically around $25 \%$ of the chord length, according to the following equation for the angle of attack.

$$
\alpha=\alpha_{0}+\alpha_{m} \times \sin \left(2 k U_{\infty} \times t / c\right)=10^{\circ}+8^{\circ} \times \sin (\omega t)
$$

Where $t$ was time in seconds, $\alpha_{O}$ was the average AoA, $\alpha_{m}$ the motion amplitude of the AoA, $k$ was the reduced frequency, $U_{\infty}$ was the freestream velocity, $c$ was the airfoil's chord length, and $\omega$ was the angular frequency of the pitching oscillation. In this study, $\alpha_{O}$ and $\alpha_{m}$ were set to $10 \mathrm{deg}$ and $8 \mathrm{deg}$, respectively. The oscillating frequency of airfoil was fixed at $2 \mathrm{~Hz}$, resulting in a reduced motion frequency $k=\pi f_{c} / U_{\infty}=0.1$, where $f$ was the physical oscillation frequency of the airfoil.

The plasma actuation was generated by an asymmetrically arranged surface DBD actuator driven by a high-voltage power supply. The DBD actuator consisted of two $0.04 \mathrm{~mm}$ thick copper tape electrodes separated by a dielectric barrier (Kapton tape) with a thickness of $0.1 \mathrm{~mm}$. The widths of the exposed and insulated electrodes were $3 \mathrm{~mm}$ and 10 $\mathrm{mm}$, respectively, and the zero gap between the two electrodes was located at $x / c=0 \%$. Thus, the total thickness of the actuator was $0.14 \mathrm{~mm}$. The actuator location can keep the actuation always occurring upstream of the separation point during the airfoil's pitching motion. The plasma actuator location and construction are shown in Fig. 1.

Pressure around the model's surface was measured by 39 pressure taps on the center of the airfoil's span, as shown in Fig. 1. Each pressure tap was connected to a fast response transducer (ENDVECO 8510B) inside the model. Each pressure transducer was in a plastic case and insulated to electric noise. The driving device, test equipment and data processing method are the same as that used in the literature [19]. Since the airfoil's AoA was changing during the experiment, it was necessary to have a high enough

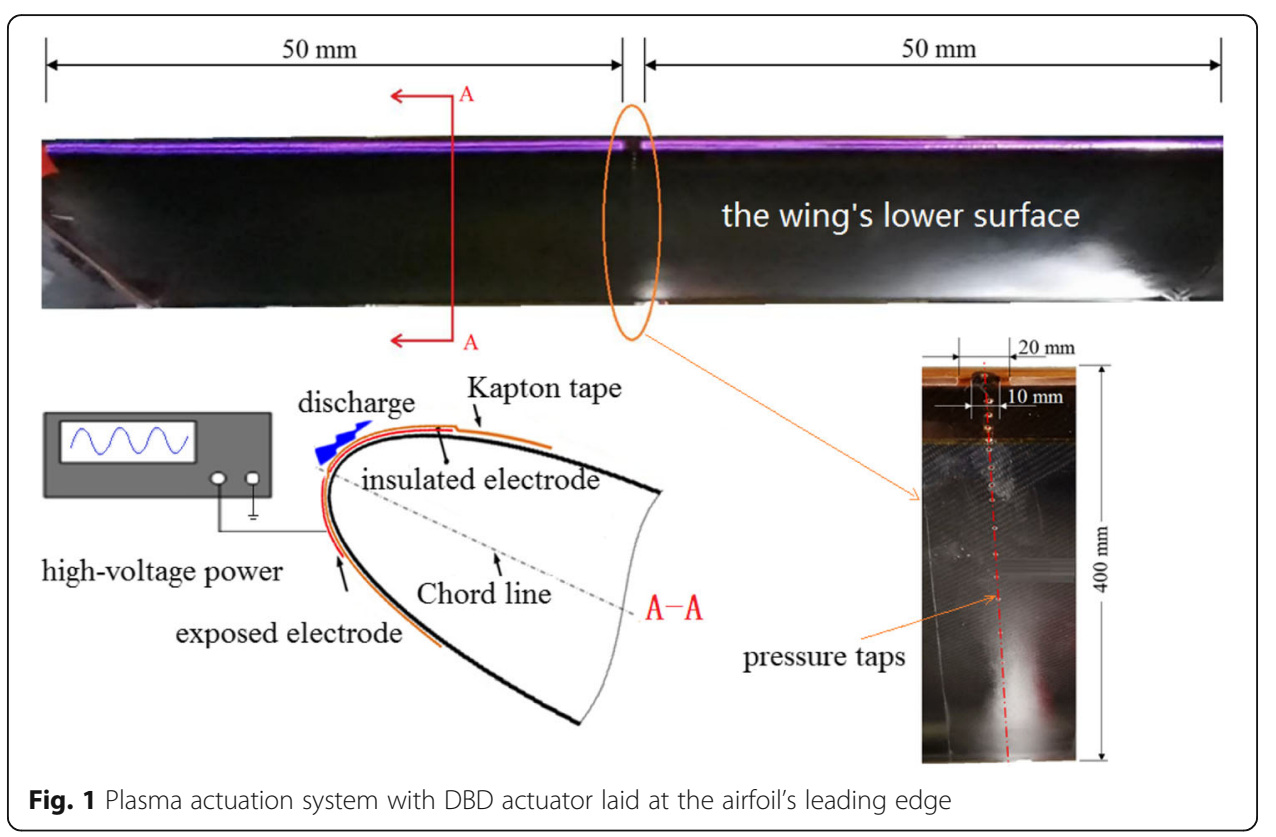


acquisition frequency during data acquisition. The dynamic pressure acquisition frequency in this article was $512 \mathrm{~Hz}$, which was 256 times the oscillation frequency of the airfoil. The pressure of each test case was obtained by phase averaging from the pressure data of 16 pitching cycles.

Pressure value $p$ at each measurement point was converted into pressure coefficient $C_{p}$ with the static pressure $p_{\infty}$ and the dynamic pressure $Q$ of the freestream.

$$
C_{p}=\left(p-p_{\infty}\right) / Q
$$

Using the phased distributions of pressure coefficient $C_{p}$, the time varying force and moment coefficients on the airfoil were obtain by integrating the pressure coefficients. The actuator near the leading edge was partly separated by the middle pressure measuring taps. Two $50 \mathrm{~mm}$-long actuators were arranged on the left and right sides of the pressure measuring taps, as shown in Fig. 1. The exposed electrodes of the two actuators were separated by a $20 \mathrm{~mm}$ wide gap, and the insulated electrodes were separated by a $10 \mathrm{~mm}$ wide gap.

The plasma actuator was connected to an alternate-current (AC) high voltage power supply with its operating frequency ranged from $6 \mathrm{kHz}$ to $40 \mathrm{kHz}$. The electrical circuit for plasma actuation system is illustrated in Fig. 1. The typical profile of discharge voltage $\left(V_{p p}\right)$-current $(I)$ signal is shown in Fig. 2 (upper). The percentage of time when the AC voltage is on is called the duty cycle (DC), which is controllable within the range of $1 \%-100 \%$. The carrier frequency of the output voltage is continuously adjustable from 6 to $40 \mathrm{kHz}$, and the modulation pulse frequency is continuously adjustable from 10 to $400 \mathrm{~Hz}$. When the pulse-modulated voltage is used to drive the DBD actuator, the actuator is cycled on and off with an unsteady period, thus, an unsteady plasma actuation is generated, with continuous vortices moving along the wall surface; When DC $=100 \%$, the actuator is driven by the continuous $\mathrm{AC}$ voltages and a steady plasma actuation can be generated, with a tangential jet along the wall [20]. The typical induced flows by steady and unsteady plasma actuation in the still air are shown in Fig. 2 (lower) [20].

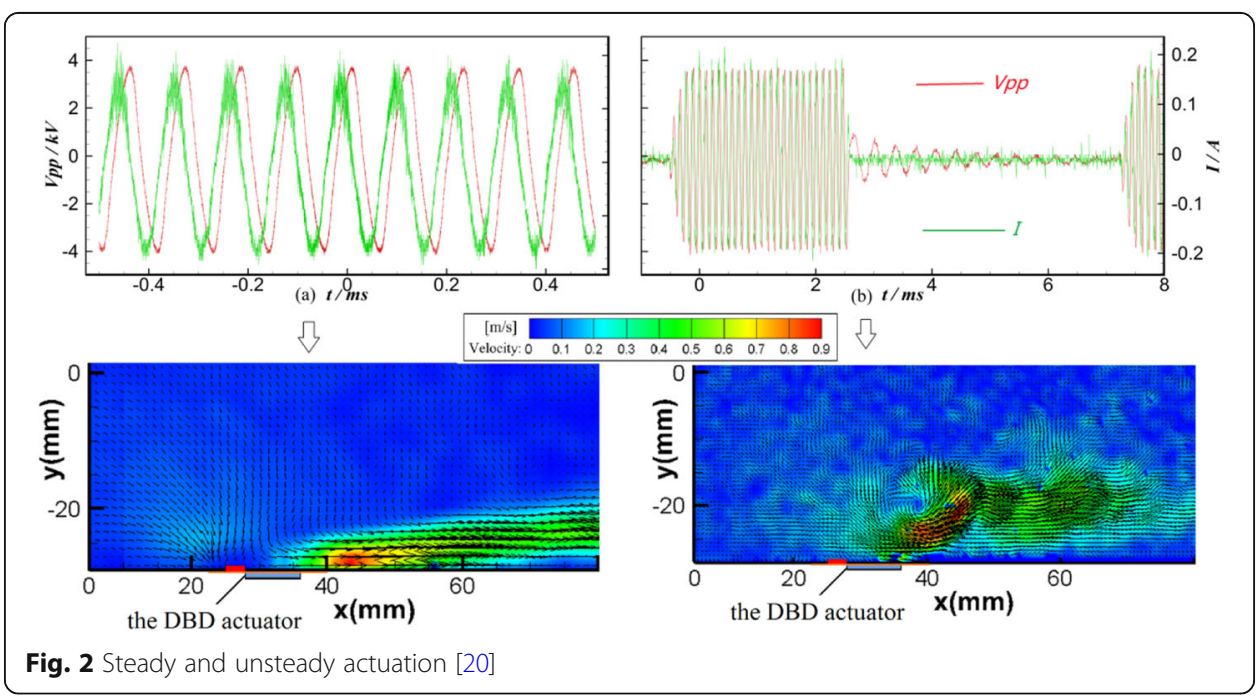




\section{Analysis of test results}

At $U_{\infty}=25 \mathrm{~m} / \mathrm{s}$, with the corresponding dynamic pressure $Q_{\infty}=341 \mathrm{~Pa}$, the critical AoA for the static airfoil was about $13^{\circ}$. In the dynamic experiments, the reduced frequency $k$ was set to 0.1 , corresponding to airfoil's oscillating frequency of $2 \mathrm{~Hz}$. The airfoil's motion was described by $\alpha=\alpha_{0}+\alpha_{m} \times \sin \left(2 k U_{\infty} t / c\right)=10^{\circ}+8^{\circ} \times \sin (12.5 t)$. Firstly, the flow control effect was assessed by using the steady plasma actuation, and then the unsteady plasma actuation was tested for comparison with the steady case. For the unsteady plasma actuation, the influence of pulse frequency $f_{p}$, duty cycle DC and voltage $V_{p p}$ on the control effect was tested to obtain the optimum parameters in the dynamic stall control.

\subsection{Improvement of aerodynamic characteristics by steady plasma actuation}

Steady actuation is a continuous discharge with a duty cycle of DC $=100 \%$. When $V_{p p}=$ $6.5 \mathrm{kV}$, three carrier frequencies of $f=7 \mathrm{kHz}, 9 \mathrm{kHz}$, and $11 \mathrm{kHz}$ were selected as the test cases. The results are shown in Fig. 3.

When the oscillating airfoil approaches its maximum AoA, the lift begins to drop and stall occurs. The lift coefficient has a large hysteresis loop. With steady actuation, the changes of lift coefficients are not obvious at $\alpha=2^{\circ}-18^{\circ}$ during the upstroke, indicating that the actuation has not achieved obvious control effect in the pitching-up phase.

The lift coefficients at $f=11 \mathrm{kHz}$ decrease slightly near the maximum AoA $(\alpha=$ $18^{\circ}$ ), while there are almost no changes for actuation at $f=7 \mathrm{kHz}$. At this up-stroke stage, we can consider that the discharge actuation has no positive control effect. At the beginning of the downstroke $\left(\alpha=18^{\circ}-15^{\circ}\right)$, there are also no obvious changes in the lift and moment coefficients. So, in the implementation of control strategy, the actuation can be turned off in the pitching-up stage to save power consumption. When the airfoil's AoA descends to a certain degree $\left(\alpha=15^{\circ}-4^{\circ}\right)$, the lift coefficients with discharge are relatively higher than that of the uncontrolled case, meaning that the lift recovery is faster and the hysteresis area decreases. Compared with the uncontrolled case, steady actuation results in an obvious decrease in the lift hysteresis area, $14 \%, 16.8 \%$ and $15.5 \%$, for the three tested carrier frequencies of $f=7 \mathrm{kHz}, 9 \mathrm{kHz}$ and $11 \mathrm{kHz}$ respectively.

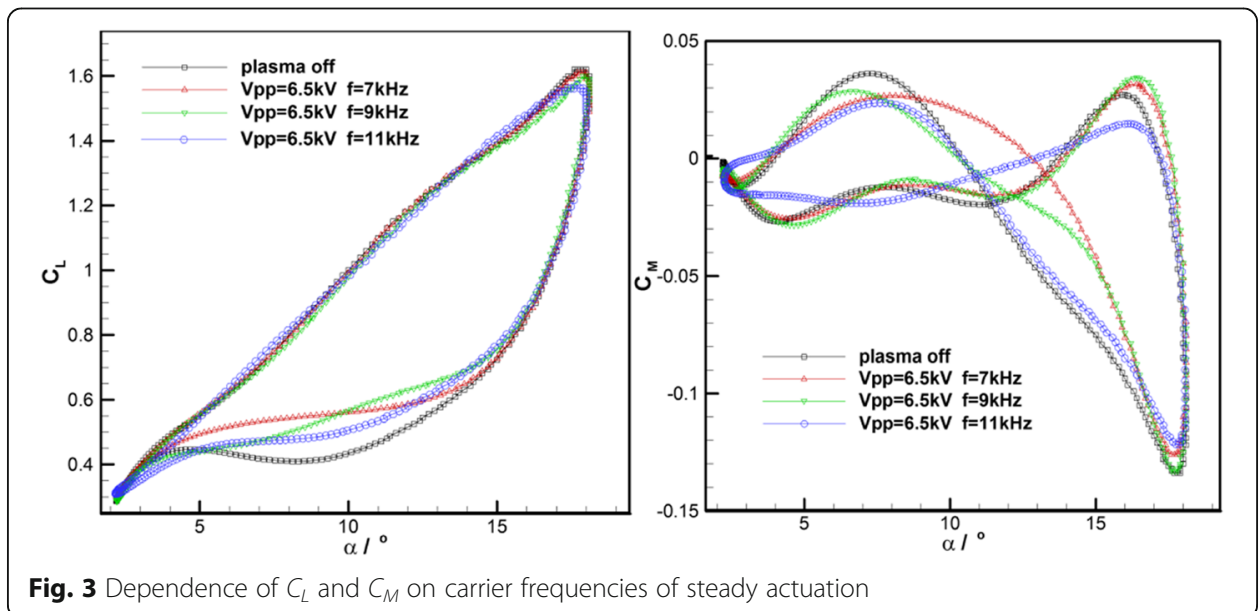


Although the reduction values of lift hysteresis area have little difference under the three carrier frequencies, the hysteresis loop's morphology is different. When the carrier frequency is higher, the lift coefficient begins to recover earlier $\left(\alpha=15^{\circ}\right.$ $\left.11^{\circ}\right)$, but then recovers $\operatorname{slowly}\left(\alpha=10^{\circ}-5^{\circ}\right)$. At a lower carrier frequency of $f=7 \mathrm{kHz}$, the lift coefficient begins to recover slowly $\left(\alpha=15^{\circ}-10^{\circ}\right)$, but then recovers quickly $\left(\alpha=10^{\circ}-5^{\circ}\right)$.

The flow's characteristic time scale over the airfoil can be defined as $T=c / U_{\infty}=$ $0.016 \mathrm{~s}$, resulting in the corresponding frequency of $62.5 \mathrm{~Hz}$, which is about two orders lower than the discharge frequency (carrier frequency). For the airfoil's oscillating frequency is just $2 \mathrm{~Hz}$, we can consider that the high-frequency discharge forms a quasi-steady disturbance during the airfoil's oscillation. Thus, the difference between $f=7 \mathrm{kHz}, 9 \mathrm{kHz}$, and $11 \mathrm{kHz}$ to the flow is that the injection energy is different. With the same actuation voltage $(6.5 \mathrm{kV})$, a higher discharge frequency means that the energy injected into flow field is larger. Literature [21] believes that when the carrier frequency is small, the volume force strength is weak. As the carrier frequency increases, the volume force gradually increases, and the power dissipation also increases; the carrier frequency continues to increase, the energy dissipation makes the air temperature rise in an advantageous position during the energy conversion process, so that the momentum exchange between the plasma and the air becomes weak, which in turn leads to a reduction in the volume force induced by DBD. For a particular actuator, there is an optimal operating frequency to maximize the volume force. This may be related to impedance matching between voltage and DBD actuator, which needs further study. But one thing is certain, that is, the increase of carrier frequency does not mean an improvement of the control effect.

For $\alpha=14^{\circ}-16^{\circ}$ of the airfoil's upstroke with no discharges, the pitching-up moment gradually increases, combined with the lift coefficient, the leading-edge suction increases more at this time, and the DSV has been formed. After $\alpha=16^{\circ}$ for the plasma off case, the moment coefficient drops sharply. With actuation, the moment stall is delayed to a certain extent and the moment begins to decrease at $\alpha=16.5^{\circ}$. After the moment coefficient reaches to its maximum value, it rebounds. Compared with the plasma off case, the moment coefficient with discharge rebounds faster. Case of $f=7 \mathrm{kHz}$ rebounds slightly faster than $f=9 \mathrm{kHz}$ case.

\subsection{Improvement of aerodynamic characteristics by unsteady plasma actuation}

When a continuous sine wave modulated by a pulsed square wave signal was applied to the DBD actuator, the unsteady actuation was formed. Here, the carrier frequency was fixed at $9 \mathrm{kHz}$ during the modulation, and the influence of pulse frequency $f_{p}$, duty cycle DC and peak to peak voltage $V_{p p}$ on the control effect was studied, to obtain the key parameters that determine the flow control effect.

\subsubsection{Influence of burst frequency}

In particular, the burst frequency or the pulse frequency, is considered to be a dominant parameter for the unsteady actuation, and is usually normalized into $F^{+}$based on the chord length $c$ and the freestream velocity $U_{\infty}$ as follows 


$$
F^{+}=f_{p} c / U_{\infty}
$$

With the actuation voltage $V_{p p}=9 \mathrm{kV}$ and the duty cycle $\mathrm{DC}=20 \%$, the burst frequency $f_{p}$ was adjusted to $62.5 \mathrm{~Hz}, 125 \mathrm{~Hz}, 250 \mathrm{~Hz}, 187.5 \mathrm{~Hz}$ and $375 \mathrm{~Hz}$, respectively. The control effect of $F^{+}=1,2,3,4$, and 6 on was investigated. The changes in lift and moment coefficients before and after actuation are shown in Fig. 4.

Compared with the plasma off case, there are no obvious changes in the lift and moment coefficients during the airfoil's pitching-up phase under actuation, with only a little decrease at the maximum AoA. The control effect is similar to that of the steady actuation cases. The most obvious changes still occur when the airfoil pitches down to a certain degree $\left(\alpha=16^{\circ}-4^{\circ}\right)$ in the downstroke stage, during which the lift coefficients can be quickly recovered. The influence of pulsed actuation frequency on the control effect is very obvious, with the area of the hysteresis loop reduced to varying degrees. With $F^{+}=1,2,3,4$, and 6 , the area of the lift hysteresis loop is reduced by $34.5 \%$, $27.5 \%, 26.3 \%, 19.9 \%$, and $21.2 \%$, respectively. From the perspective of the lift hysteresis loop, the optimal pulse actuation frequency is $F^{+}=1$, which is consistent with the law of static airfoil's separation control at high angles. From the changes of the moment coefficients, the moment stall under pulsed actuation is not improved effectively. The maximum nose-down moment coefficient is slightly reduced. After descending to the maximum nose-down moment coefficient, the rapid rebound of moment coefficients after pulsed actuation is very obvious, indicating that the leading-edge suction of the airfoil is quickly enhanced.

At $\alpha=13^{\circ}$ on upstroke and downstroke, the changes of pressure coefficient before and after control are compared, as shown in Fig. 5. At $\alpha=13^{\circ}$ of the upstroke, the airfoil's pressure distributions of the tested cases are almost same without any significant difference. At this point, the leading edge has an obvious suction. While at $\alpha=13^{\circ}$ of the downstroke for the plasma off case, the pressure distribution of the plasma off case is nearly flat and the suction force on the leading edge is relatively small. Correspondingly, the lift coefficient is low $\left(C_{L}=0.57\right)$ and the nose-down moment coefficient is still large $\left(C_{M}=-0.048\right)$. After actuation, the leading-edge pressure shows a significant negative pressure peak with $C_{p}$ increased from -1 to -3.5 . The reverse pressure gradient of the upper surface is obvious, and the separation is effectively controlled at this point.

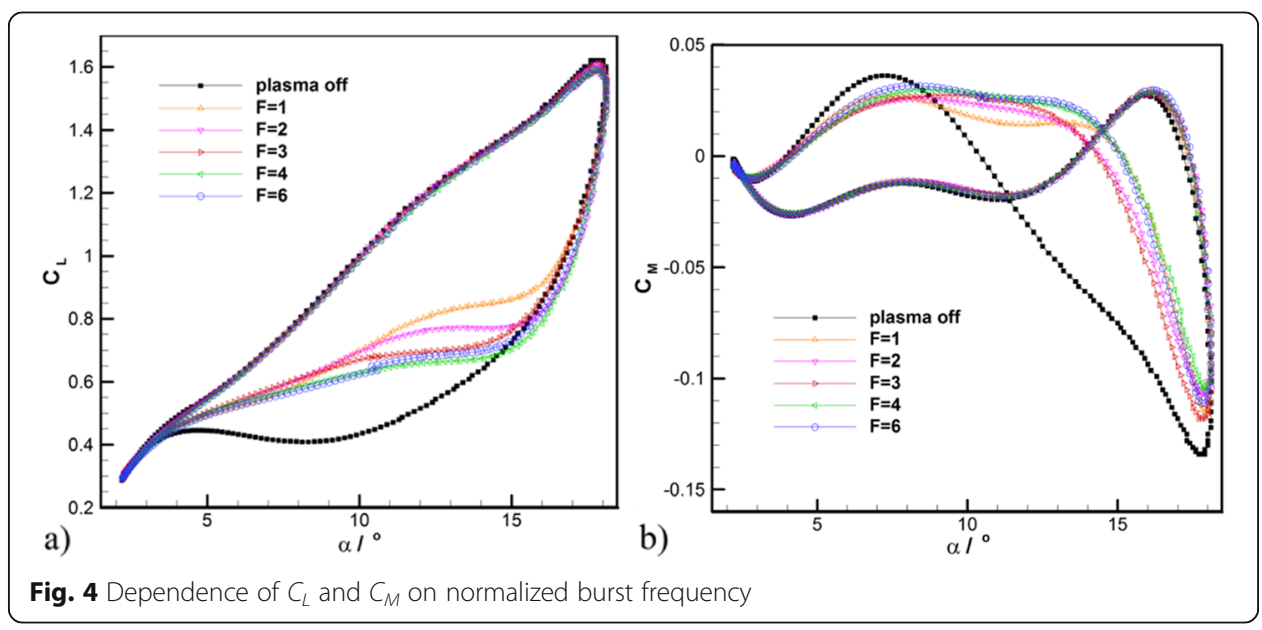




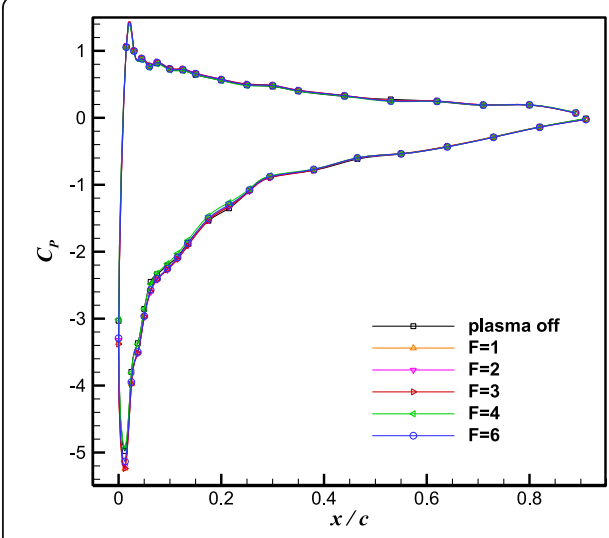

a) $\alpha=13^{\circ}$ on the upstroke

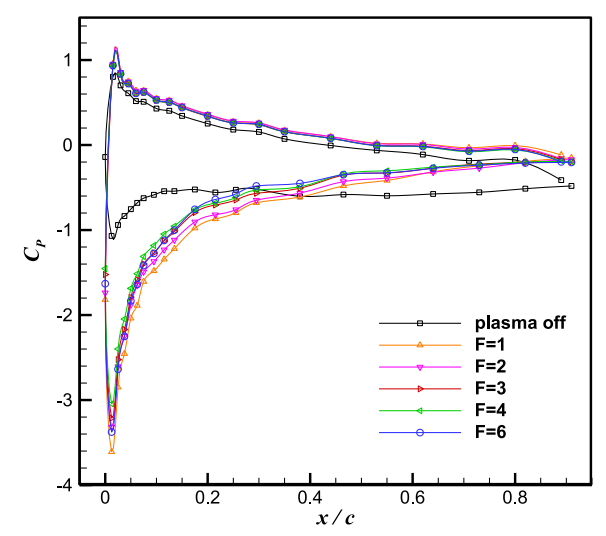

b) $\alpha=13^{\circ}$ on the downstroke

Fig. 5 The effect of normalized burst frequency on the airfoil's pressure distribution of $a=13^{\circ}$

\subsubsection{Influence of duty cycle}

In addition to the burst frequency, the duty cycle is also an important parameter of the unsteady actuation. The smaller the duty cycle, the stronger the non-stationarity of the actuation. On the contrary, the larger the duty cycle, the more stable and steady of the actuation. When the duty cycle is $99 \%$, it can already be considered as a steady actuation. With the normalized burst frequency of $F^{+}=2$ (carrier frequency $f=9 \mathrm{kHz}$ ) and actuation voltage of $V_{p p}=8 \mathrm{kV}$, the effect of the duty cycle with DC $=10 \%, 20 \%, 40 \%$, $60 \%, 80 \%$ on the control was tested. The changes of the lift coefficients and moment coefficients before and after actuation are shown in Fig. 6.

The effect is more obvious at $\alpha=15^{\circ}-5^{\circ}$ in the downstroke stage than other AoA ranges, and the lift coefficient under actuation recovers faster compared with the plasma off case, which is similar to the control effects in Fig. 3 and Fig. 4. The actuation at $\mathrm{DC}=10 \%, 20 \%, 40 \%, 60 \%$, and $80 \%$ duty cycles can reduce the hysteresis loop area of lift coefficients by $33.2 \%, 25.5 \%, 25.9 \%, 24.3 \%$, and $16.1 \%$, respectively. Overall, in the tested duty cycle values, the smaller the duty cycle, the greater the reduction in the hysteresis loop area. The actuation at DC $=20 \%, 40 \%, 60 \%$ has no obvious difference in the changes of lift and moment coefficients. When DC $=80 \%$, the reduction of

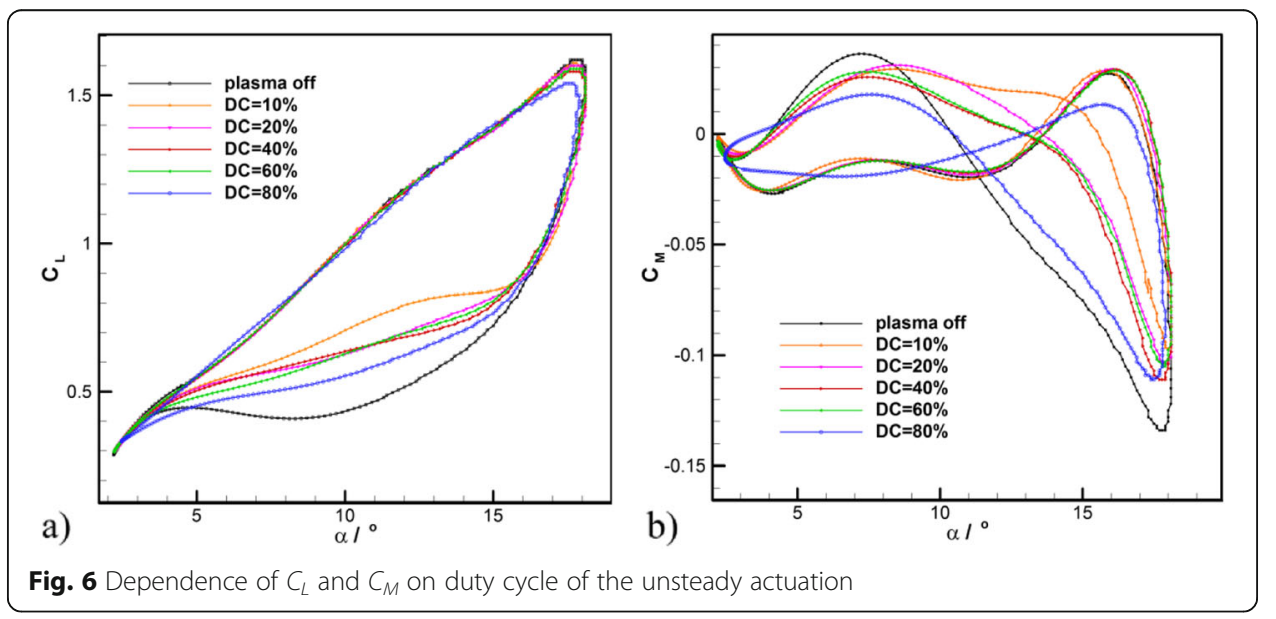


hysteresis loop area is about $15 \%$, close to that of the steady actuation in Fig. 3. For the tested duty cycles, actuation at DC $=10 \%$ obtains the best control effect, which means that the short-pulse dischargeshave a better impact on the flow field.

In Fig. 6b, the maximum nose-down moment is also reduced under actuation. With actuation, the moment coefficient recovers faster after the maximum nose-down moment. When $\alpha=17^{\circ}$ at the downstroke, although the corresponding lift coefficient does not change much, the moment has begun to recover quickly, indicating that the pressure distribution has been improved with the leading-edge suction enhanced and the nose-down moment changed.

At $\alpha=13^{\circ}$ on upstroke and downstroke, the changes of pressure coefficient with and without actuation are compared, as shown in Fig. 7. During the pitching-up phase, the pressure distributions under actuation at different duty cycles have a good repeatability, consistent with the lift coefficients with no obvious difference in this stage. During the pitching-down phase, for the plasma off case and actuation at $\mathrm{DC}=80 \%$ case, the pressure of the airfoil's upper surface is relatively flat, and the leading-edge suction is small, indicating that there is still a serious flow separation near the leading edge. When the actuator works at DC $=10 \%, 20 \%, 40 \%$ and $60 \%$, the leading-edge suction is significantly enhanced, especially when DC $=10 \%$, indicating that the flow is reattached.

This conclusion is very meaningful to guide the next research, and the development of short pulse actuation has more advantages, such as unsteady nanosecond pulse DBD or microsecond pulse DBD.

\subsubsection{Influence of voltage}

By changing the actuation voltage $V_{p p}$ to 8,9 , and $10 \mathrm{kV}$ with its pulsed actuation frequency fixed at $F^{+}=2$, the influence of the actuation voltage on the control effect was studied. The changes of the lift and moment coefficients before and after actuation are shown in Fig. 8. The voltage has a greater influence on the hysteresis loop of the lift coefficients. The discharges at $V_{p p}=8,9$, and $10 \mathrm{kV}$ reduced the area of the lift hysteresis loop by $26.4 \%, 33.1 \%$, and $32.2 \%$, respectively. Similar to the above results, different

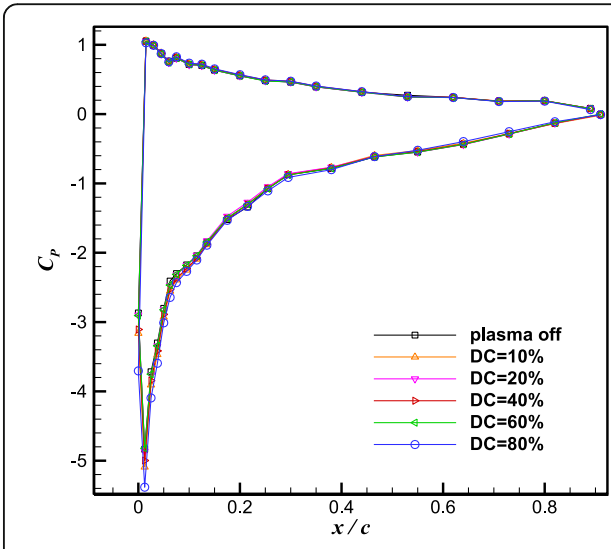

a) $\alpha=13^{\circ}$ on the upstroke

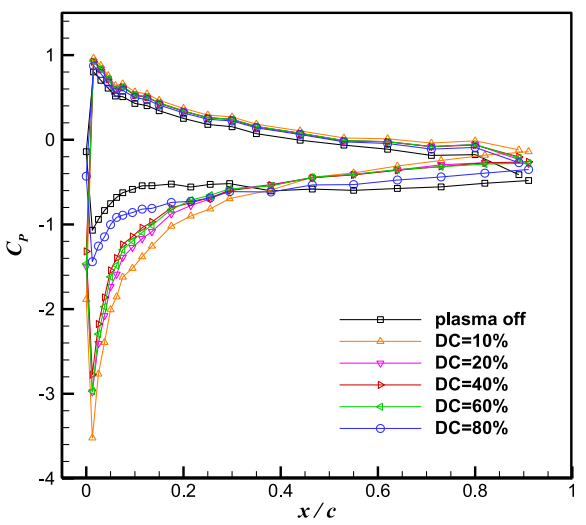

b) $\alpha=13^{\circ}$ on the downstroke

Fig. 7 The effect of duty cycle on the pressure coefficient of $a=13^{\circ}$ 

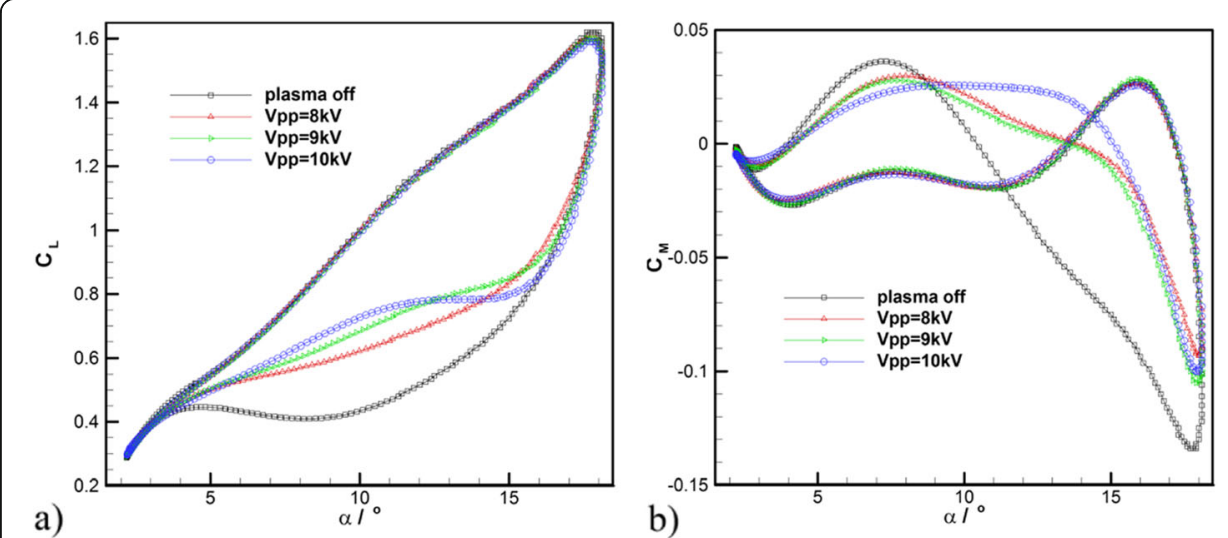

Fig. 8 The effect of actuation voltages on the lift and moment coefficients

actuation voltages did not improve the lift and moment coefficients during the pitching-up phase. When the airfoil drops below $15^{\circ}$ from its maximum AoA, the lift coefficient recovers faster. A higher voltage means greater pulsed actuation intensity, yielding stronger flow control ability, and finally the lift coefficient recovers faster. The corresponding moment coefficient shows the same control law.

The changes of pressure coefficients before and after discharges at $\alpha=11^{\circ}$ were shown in Fig. 9. When $\alpha=11^{\circ}$ in the pitching-up stage, the controlled cases under different actuation voltages have a good coincidence with the plasma off case. Correspondingly, there is no obvious control effect on the lift and moment coefficients in the pitching up phase. For the plasma off case at $\alpha=11^{\circ}$ on downstroke, the suction peak at the leading edge is not large enough, indicating that there is a severe flow separation at the leading edge. After actuation, the suction of the leading edge is obviously enhanced. The greater the voltages, the larger the suction of the leading edge, which is consistent with the change of lift coefficient depicted in Fig. 8.

\section{Mechanism analysis}

From the above results of dynamic stall control, the AC DBD actuation cannot delay the dynamic stall AoA, and the control effect at high AoA is also limited. Post and

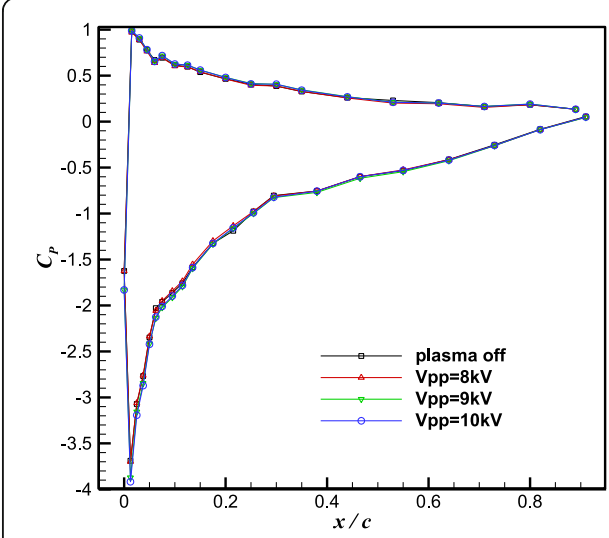

a) $\alpha=11^{\circ}$ of the upstroke

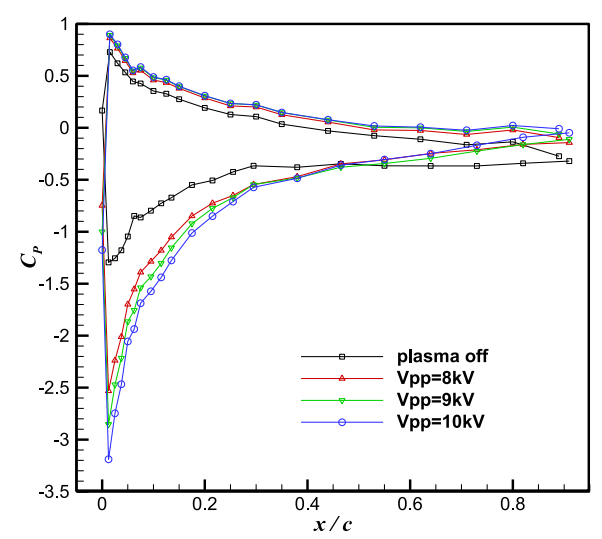

b) $\alpha=11^{\circ}$ of the downstroke

Fig. 9 The effect of actuation duty cycle on the pressure coefficient 
Corke [7] showed similar results that the plasma actuator enhances the lift force during pitching down rather than pitching up. Paper [12] conducted the time-resolved PIV measurement to understand the flow control mechanism by the plasma actuator, and found that the clear vortices appeared at the leading edge bring entrainment of main flow. With the flow conditions being the same as that of the experiment in Ref. [12], the LES result in Ref. [22] also showed a large leading-edge vortex emanates after every plasma burst actuation, which was not observed for the plasma off case. These plasmainduced large vortices not only induced suction force but also promoted reattachment for exchanging the momentum between freestream and separated boundary layer at the last phase of the pitching down. Combined with the above references, we discussed the effectiveness of the plasma actuator on the dynamic flowfields as follows.

At high AoA during the pitching up phase, due to the effect of downwash flow and the DSV, the dynamic stall AoA is much larger than the static stall AoA (generally larger than $5^{\circ}$ ), and the corresponding lift coefficient is also higher than the maximum lift coefficient in the static situation. However, when the DSV moves downstream over the 0.25 chord length, the nose-down moment increases, and the moment stall occurs; when the DSV is removed from the airfoil, the lift dynamic stall occurs. At this moment, the large AoA and the large separation angle of the leading-edge shear layer result in a large reverse pressure gradient; this may exceed the control capability of AC DBD actuation. At this time, the flow field before and after actuation can be depicted in Fig. 10a and b, which are extracted from Ref. [12] and Ref. [15]. Although AC DBD produces a certain disturbance and induces the formation of a spanning vortex, the vortex will be swallowed by the stronger separation vortex, as shown in Fig. 10b, msaking the stall state unable to be controlled effectively.

When the airfoil starts pitching down from its maximum AoA, the effect of upwash flow is gradually obvious. The effective AoA is still large, and the separation angle of the separated shear layer at the leading edge is still large. It is still difficult to control the flow separation for the reverse pressure gradient is large, and the flowfield after actuation is similar to Fig. 10b.

Only when the effective AoA pitches down to a certain extent, the separation angle of the leading-edge shear layer becomes small, and the reverse pressure gradient is moderated, then, the AC DBD actuation can induce the formation of a closed span vortex which can be locked on the airfoil surface, as shown in Fig. 10c. At this moment, AC DBD actuation can play an effective role in controlling the flow separation.

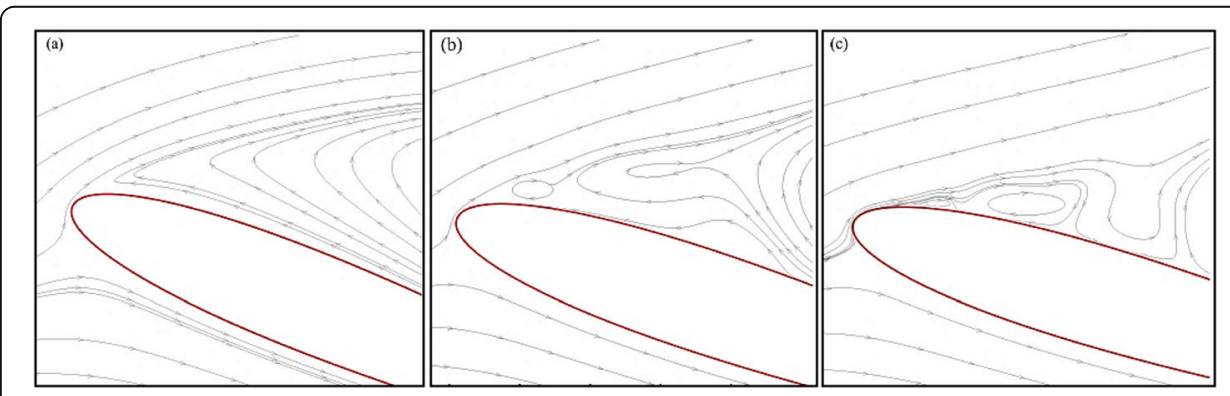

Fig. 10 The Schematic diagram of flow field before and after actuation in dynamic stall state 
Therefore, the flow control effect of dynamic stall by AC DBD actuation is strongly dependent on the history of the airfoil's AoA.

\section{Conclusions}

This study extends the DBD plasma actuation to the dynamic stall control of an advanced rotor airfoil, verifies the effectiveness of AC DBD actuation to reduce the area of lift hysteresis loop of the oscillating airfoil, and studies the influence law of plasma actuation parameters to the control effect. The main conclusions are as follows:

(1) At the freestream velocity of $25 \mathrm{~m} / \mathrm{s}$ and Reynolds number of $5.2 \times 10^{5}$, the AC DBD plasma actuation can effectively reduce the area of the lift hysteresis loop of an oscillating airfoil. Unsteady plasma actuation has obvious advantages in dynamic stall control. The unsteady actuation can reduce the area of lift hysteresis loop by more than $30 \%$, while the reduction is less than $20 \%$ for steady actuation.

(2) For the unsteady AC DBD plasma actuation, the smaller duty cycle can obtain more obvious control effect; the optimal unsteady reduced actuation frequency $F^{+}=1$; the larger the actuation voltage, the faster the recovery of lift and moment coefficients. This conclusion is meaningful to guide the next research. The development of short pulse actuation has more advantages in dynamic stall control, such as unsteady nanosecond pulse DBD or microsecond pulse DBD.

(3) The flow control effect of dynamic stall is strongly dependent on the history of AoA. Plasma actuation does not improve the lift coefficients of the entire upstroke and the initial downstroke phase. Only when the effective AoA drops to a certain degree, AC DBD actuation can play an effective role and make the lift coefficient recover quickly.

Finally, the flow control mechanism is discussed based on international numerical simulation and flow field test. That the spanwise vortex induced by plasma excitation is locked on the airfoil is key to effectively controlling the separated flow. The formation and development of spanwise vortex are strongly dependent on the Angle of attack. In this paper, the relevant numerical simulation and the flow field measurement have not been carried out, so the control mechanism needs further study.

\section{Acknowledgements}

The authors would like to thank Chen Lei of China Aerodynamics Research and Development Center for his active cooperation in the preparation and implementation of the experiment. Thank Associate Professor Liang Hua of Air Force Engineering University for the plasma power supply.

Authors' contributions

The contribution of the authors to the work is equivalent. All authors read and approved the final manuscript.

\section{Funding}

This work is supported by the Open Fund from State Key Laboratory of Aerodynamics of China (Grant No. SKLA20180207) and Feng Lei Youth Innovation Fund of CARDC (Grant No. PJD20190003). This work is also supported by National Numerical Wind tunnel project (NNW2018-ZT3B08)

Availability of data and materials

The datasets used or analyzed during the current study are available from the corresponding author on reasonable request. 


\section{Author details}

'State Key Laboratory of Aerodynamics, China Aerodynamics Research and Development Center, Mianyang 621000,

China. ${ }^{2}$ Low Speed Aerodynamics Institute, China Aerodynamics Research and Development Center, Mianyang 621000

China. ${ }^{3}$ Science and Technology on Plasma Dynamics Laboratory, Air Force Engineering University, Xi'an 710038, China.

Received: 21 October 2020 Accepted: 17 January 2021

Published online: 02 March 2021

\section{References}

1. Hardy P, Barricau P, Belinger A et al (2010) Plasma synthetic jet for flow control. In: Proceedings of the 40th Fluid Dynamics Conference and Exhibit; 28 June -1 July 2010. AIAA, Chicago

2. Jin D, Li YH, Jia M et al (2014) Investigation on the shockwave induced by surface arc plasma in quiescent air. Chin Phys B 23:396-403

3. Wang J, Li YH, Xing F (2009) Investigation on oblique shock wave control by arc discharge plasma in supersonic airflow. J Appl Phys 106(7):073307

4. Benard N, Moreau E (2014) Electrical and mechanical characteristics of surface AC dielectric barrier discharge plasma actuators applied to airflow control. Exp Fluids 55(11):1846

5. Durasiewicz C, Singh A, Little J (2018) A comparative flow physics study of Ns-DBD vs Ac-DBD plasma actuators for transient separation control on a NACA 0012 airfoil. In: Proceedings of the 2018 AlAA Aerospace Sciences Meeting; 8-12 January 2018. AlAA, Kissimmee, Florida

6. Wang JJ, Choi KS, Feng LH et al (2013) Recent developments in DBD plasma flow control. Prog Aerosp Sci 62:52-78

7. Post ML, Corke TC (2006) Separation control using plasma actuators: dynamic stall vortex control on oscillating airfoil. AIAA J 44:3125-3135

8. Starikovskiy A, Meehan K, Miles R (2018) Dynamic stall control by NS SDBD actuator. In: Proceedings of the 2018 AIAA Aerospace Sciences Meeting; 8-12 January 2018. AlAA, Kissimmee, Florida

9. Phan MK, Shin J (2016) Numerical investigation of aerodynamic flow actuation produced by surface plasma actuator on 2D oscillating airfoil. Chin J Aeronaut 29(4):882-892

10. Yang HS, Zhao GY, Liang $\mathrm{H}$ et al (2020) Research progress on influence factors of airfoil dynamic stall flow control. Acta Aeronaut Astronaut Sin 41(8):023605

11. Chen WX (2008) Dynamic stall simulation in CFD. Helicopter Technique 155(3):55-68

12. Mitsuo K, Watanabe S, Atobe T et al (2013) Lift enhancement of a pitching airfoil in dynamic stall by DBD plasma actuators. In: Proceedings of the 51st AIAA Aerospace Sciences Meeting including the New Horizons Forum and Aerospace Exposition; 07-10 January 2013. AIAA, Grapevine (Dallas/Ft. Worth Region), Texas

13. Frankhouser M, Hird K, Naigle S et al (2015) Nanosecond dielectric barrier discharge plasma actuator flow control of compressible dynamic stall. In: Proceedings of the 46th AIAA Plasmadynamics and Lasers Conference; 22-26 June 2015. AIAA, Dallas, TX

14. Sekimoto S, Fukumoto H, Shimomura S et al (2018) Experimental analysis of burst actuation for separation control around a pitching NACA0015 airfoil using a DBD plasma actuator at low Reynolds number. In: Proceedings of the 2018 AIAA aerospace sciences meeting; 8-12 January 2018. AIAA, Kissimmee, Florida

15. Yu HC, Zheng JG (2020) Numerical investigation of control of dynamic stall over a NACA0015 airfoil using dielectric barrier discharge plasma actuators. Phys Fluids 32(3):035103

16. Iwasaki Y, Nonomura T, Nankai K et al (2020) Dynamic stall control around practical airfoil using nanosecond-pulsedriven dielectric barrier discharge plasma actuators. Energies 13(6):1376

17. Fukumoto $\mathrm{H}$, Aono $\mathrm{H}$, Nonomura T, et al (2018) Large-eddy simulations of flow control effects of a DBD plasma actuator at various burst frequencies on a dynamic flowfield around a pitching NACA0012 airfoil at Reynolds number of 256,000. 2018 AIAA Aerospace Sciences Meeting; 8-12 January 2018. AIAA, Kissimmee. https://doi.org/10.2514/6.2018-1550

18. Lombardi AJ, Bowles PO, Corke TC (2013) Closed-loop dynamic stall control using a plasma actuator. AIAA J 51(5):1130-1141

19. Li GQ, Chang ZQ, Zhang X et al (2018) Experiment on flow control of airfoil dynamic stall using plasma actuator. Acta Aeronaut Astronaut Sin 39:122111 (in Chinese). https://doi.org/10.7527/S1000-6893.2018.22111

20. Han MH (2015) Study on plasma flow control of flying wing and lifting device. Dissertation, Air Force Engineering University

21. Corke TC, Post ML, Orlov DM (2007) SDBD plasma enhanced aerodynamics: concepts, optimization and applications. Prog Aerosp Sci 43(7-8):193-217

22. Fukumoto $\mathrm{H}$, Aono $\mathrm{H}$, Tanaka $\mathrm{M}$ et al (2016) Control of dynamically stalled flowfield around a pitching airfoil by DBD plasma actuator. In: Proceedings of the 8th AIAA Flow Control Conference; 13-17 June 2016. AIAA, Washington, D.C.

\section{Publisher's Note}

Springer Nature remains neutral with regard to jurisdictional claims in published maps and institutional affiliations. 